\title{
An Operational Framework for an Online Community Based on the Commons, Dissensus and Shared Knowledge
}

Hülya Ertas(1), Burak Pak(1) and Caroline Newton(1)(2)

(1)KU Leuven Faculty of Architecture, Campus Sint-Lucas, Rue des Palais 65, 1030 Brussels, Belgium

(2)TU Delft Department of Urbanism, Faculty of Architecture and the Built Environment, Julianalaan 134, 2628 BL Delft, The Netherlands

\begin{abstract}
In this article we introduce a dissensual knowledge production mechanism for the creation and governance of the commons. Rather than seeking a singular truth which is a social construct, producing a bricolage of truths by citizen associations or communities opens up new perspectives for the commons and democracy. Taking an online community as a case study we analyze knowledge production and sharing mechanisms, and conflict generation and resolution processes, using simple statistics, text mining, disagreement hierarchy, community guidelines and group rules. We use the conclusions drawn from these techniques in the Institutional Analysis and Development framework. Superposing our findings and a critical reading of current best practices we define the four main principles as the operational framework for an online community based on the commons, dissensus and shared knowledge: distributed versioning, layering, inclusion and self-determination.
\end{abstract}

Keywords: commons, dissensus, shared knowledge, p2p, online community

\section{Introduction}

Social media (e.g., Facebook, Twitter) and online forums (e.g., Reddit, Discourse) allow people from around the world to discuss their political views and their concerns about societal issues. These platforms act as knowledge hubs and shape the views of online communities, independently from their countries of origin. From alt-right groups to the propagators of postcapitalism we can detect a wide range of political perspectives within knowledge-sharing spaces. Building on Blommaert (2016) we argue that these platforms and associated online communities are constructing bricolages of 'truths'. During the process of bricolage conflicts occur naturally because truth, as a social construct, can have multiple facades. Starting with Weber's criticism of objectivity in social science, philosophers such as Nietzsche, Lyotard, Derrida, Heidegger and Foucault made significant efforts to dismantle the idea of factual truth (Angermuller 2018), discussed its unreliability, and even invented concepts to replace it (McIntryre 2018).

In this article we analyze how online groups construct shared knowledge and explore how divergent and alternative voices are allowed to coexist in these groups. Inclusion of voices, ideas and knowledges of marginal groups is beneficial for society as a whole (Foucault 1972 and 1982); allowing disagreement and oppositional ideas enables emancipation. We argue that online platforms function as well-organized citizens' associations that can play an important role in helping to inform citizens and allowing people to 'create centers of political power independent of the state' (Klein 1999: 213). According to deTocqueville,

When the members of a community are forced to attend to public affairs, they are necessarily drawn from the circle of their own interests, and snatched at times from self-observation. As soon as a man begins to treat 
of public affairs in public, he begins to perceive that he is not so independent of his fellow-men as he had at first imagined, and that, in order to obtain their support, he must often lend them his co-operation. (deTocqueville 1863: 124-125)

These associations allow for the (political) education of citizens. Horton and Freire (1990: 182-185) discuss how education, which is different from schooling, has an emancipatory potential. If used wisely, that potential allows organizations already fighting the status quo to push society towards real structural change. For this to happen educators need to create the right environments and conditions to allow students to become themselves.

Furthermore, Freire emphasizes that 'the educator also has the duty of not being neutral, (...). The educator as an intellectual has to intervene. He cannot be a mere facilitator. He has to affirm to himself or herself' (Horton and Freire 1990: 181). This conceptualization is directly applicable in the context of online platforms that support people's (self-)education and networks for sharing ideas and constructing knowledge. It is especially relevant in networks that advocate alternative social organizations open to everyone and based on the concept of the commons.

This article shows how the bricolage of truths emerges within an online community but is not explicitly recognized by it. We then propose a conceptual basis for a novel online platform that facilitates dissensual knowledge production to address the issues of conflict resolution, self-governance and democracy. The article critically examines the construction of truths and seeks a novel methodology for building and sustaining online communities using three central concepts: commons, dissensus, and shared knowledge. It unpacks social practices which govern the construction of truths on the platform using Gielen's (2018) understanding of the commons as a 'heterotopian environment where conflicting interests intersect'. Refusing to boil down community discussions into a singular truth, we elaborate how the practices of commoning may embrace a bricolage of truths. This does not necessarily imply production, reproduction or dissemination of post-truth, but signals an opening which allows for dissensus. Building on Rancière (2010), dissensus allows us to cut across the truths of different voices in online communities to introduce other truths in these different frames of reference.

This approach contrasts heavily with the current conflict management practices in online communities, whereby each interlocutor makes concessions to reach a middle ground, and which is an adaptation of the neoliberal managerial logic to self-organization. In these mainstream consensus-seeking process, shared knowledge is funneled down to a singular truth, in turn limiting not only knowledge sharing, but also wider contributions of the community. In contrast, dissensus mechanisms allow multiplication, amplification and forking of truths, thus enabling the emergence of critical knowledge commons.

The article starts by constructing a theoretical framework that connects the concepts of dissensus, the commons and shared knowledge, setting up a framework for discussion and action. Using this framework, we analyze an online community centered around the practices of sharing and the commons: the P2P Facebook group with 6,371 members. This analysis focuses on protocols and (non)hierarchies for sharing and editing knowledge and managing conflict. Synthesizing our theoretical study and practical analyses, we propose a set of principles for a novel online community based on dissensus, the commons and shared knowledge.

\section{Commons, Shared knowledge and Dissensus}

\section{Commons}

The popularity of the commons has risen steeply in recent decades, especially since the 
recognition of Elinor Ostrom's work in 2009 when she received the Nobel Prize in economics. Her detailed fieldwork studies the sharing of natural resources and leads to an extensive and comprehensive theory of the commons. Ostrom shows that there is an alternative to the neoliberal market-oriented management of resources. Already in her seminal work Governing the Commons. The Evolution of Institutions for Collective Action Ostrom (1990) stresses that the governance of the commons is crucial. She implicitly acknowledges that the notion of the commons goes beyond the material, and is about organization and governance of access to materials or assets (Deleixhe 2018; Euler 2018).

Building on the works of DeAngelis and Stravides (2010), Bodirsky (2018), Deleixhe (2018), Euler (2018), Susser (2017a and 2017b) we do not provide one unifying definition of 'the commons'. Instead, we discuss a framework that helps to understand the different elements that constitute this key concept. Commons are asset-based, and assets can be material (e.g. water) and intangible (e.g. knowledge, cultural practices). Commons are initiated and sustained by a group of people, often referred to as 'commoners', who form a community that designs rules and procedures to organize and govern the shared resources. These groups are not necessarily bounded by physical territory or homogeneous. Through a process of commoning, the commons are sustained and reproduced by the members of the community. De Angelis and Stravides (2010) point out that every community is made up of subgroups and individuals, all with their own norms, values, cultures and connections. Consequently, different opinions and viewpoints will always be present in a community. These differences increase in online communities, which are composed of people from around the world. If they want to function as commons, online communities need to incorporate heterogeneity and include non-dominant voices.

At least since Ostrom (1990), the notion of the commons is seen as an alternative for the organization and structure of society. Where Ostrom (1990) discusses the commons along with the state and the market, other authors have quickly seen the commons as a potential counter-hegemonic force, challenging the current neoliberal capitalist organization of our societies and thus pulling the commons to the political level. For Dardot and Laval (2018: 22) 'a commons are first and foremost an institutional affair.' They continue: 'The very act of establishing a common is in and of itself a democratic act. Democracy is, in essence, coparticipation in public affairs' (2018: 22-23). Dardot and Laval see a cry for the renewal of real democracy in movements such as Occupy Wall Street, the Indignados, and others. It is the engagement in the commons, the act of commoning, that constitutes the commons. For Dardot and Laval, it is this far reaching self-government practice of commoning that holds the promise to resuscitate democracy. When discussing the commons, Dardot and Laval and many others assume that self-governance happens in a harmonious and cooperative atmosphere (Deleixhe 2018). However, our experiences in this research acknowledge warnings raised by Deleixhe (2018) and others (Bodirsky 2018; Susser 2017a and 2017b) that power inequalities and forms of oppression are present within the commons, but that they are too often ignored or hastily resolved, thus leading to a false feeling of harmony and democracy.

Building on the work of Chantal Mouffe and Martin Deleixhe we argue that introducing an agonistic model which recognizes the value of conflict and dissensus might help us to enhance the democratic potential of the commons and allow groups to take stronger positions beyond their own politics. An agonistic model allows groups to be more attentive to the risk of creating their own echo chambers and stand stronger in the public arena of the web, using temporary coalitions to enhance the possibility of resistance against the dominant neoliberal model, thus also resisting the treat of enclosure of the commons and its private appropriation. 


\section{Dissensus}

According to Mouffe (2018: 93), 'agonistic confrontation, far from representing a danger to democracy, is in reality the very condition of its existence. (...) democracy (...) must also enable the agonistic expression of conflict, which requires that citizens genuinely have the possibility of choosing between real alternatives.' Dissensus allows differences to exist. It calls the obvious and the given into question, thus opening up new possibilities (Rancière 2009: 49). Deleixhe portrays these commons as 'harmonious self-governing schemes of cooperation' (2018: 66). This naive view neglects Ostrom's emphasis on the need for conflict resolution mechanisms, as they are an indication of differences of opinion within the commons. The false belief in 'one community' is very strong and needs to be deconstructed to allow for strong counter-hegemonic coalitions. In opposition to quick conflict resolution which safeguards the false image of harmonious commons, we stress the need for the inclusion of mechanisms and protocols that incorporate different voices.

Mouffe's concept of dissensus offers a strong foundation for the construction of these protocols. She understands conflict as a constructive force that helps people to move away from a non-productive consensus and supports status quo. Mouffe's approach stresses the 'maximum autonomization' (Laclau and Mouffe 1985: 167) of individuals or groups, whereby each political project is acknowledged in its own respect. In this model, 'the transformation of political enemies into adversaries, respecting the other within the necessarily conflictual democratic framework' (Carpentier and Cammaerts 2006: 965) allows co-existence of and debate between different viewpoints and opinions.

\section{Commons-based Peer Production of Shared Knowledge}

Open source production has emerged as a collective practice with novel, sophisticated and structured methods for collaboration. Common principles and characteristics of social production of knowledge shared by numerous open source platforms such as Kuro5hin, Github, Slashdot and Wikipedia are: (a) decentralization, (b) the use of social cues and motivations, (c) modularity enabling incremental and asynchronous collaboration, (d) heterogenous granularity and (e) low-cost (in terms of time, usability and finances) integration of the modules (Benkler and Nissembaum 2006). These are made available through a Version Control System (VCS) that allows participants to keep track of and manage the modifications of the common source over time. This can be organized in at least two different ways: centralized versioning and decentralized versioning (Figure 1).

Wikipedia uses centralized version control for each language group, thus enforcing shared knowledge production to reach consensus - a single truth. Centralized versioning leads to conflicts that result in the creation of 'protected' and 'semi-protected' pages editable by only a limited number of users such as administrators or registered contributors. All content is stored in centralized servers that are inaccessible to ordinary users. As opposed to Wikipedia's consensus-based collaboration, the Linux open source community supports a distributed control versioning system (Git) for collaborative software development (Torvalds 2007). Git enables non-linear co-creation of source code in virtually unlimited number of parallel branches through a complex collaboration model known as the 'Git way of working' (Chacon and Straub 2014). This mode of collaboration facilitates a multi-versional representation of content as a range of snapshots. These snapshots are updated every time a user saves a different state of source code.

Content is stored in three forms in the Git distributed control versioning system: committed (stored locally), modified (updated but not committed), and staged (marked for the next commit). These forms of content travel between three different parts of the Git project: the directory, the working directory and the staging area. This complex structure enables each 
user to create an alternative repository and store it locally on their server or on their personal computer. Git provides full rights to the users to access content, review updates by other users, reveal conflicts, discuss issues and address conflicts, and create and store local versions, which helps constitute a relatively horizontal power structure. Distributed VCS allows the construction of different realities and results in the creation of a plethora of alternatives. Although this system is currently used to produce software, it may be used for operationalizing dissensus in commons-based peer production of shared knowledge as a means for generating a bricolage of truths.
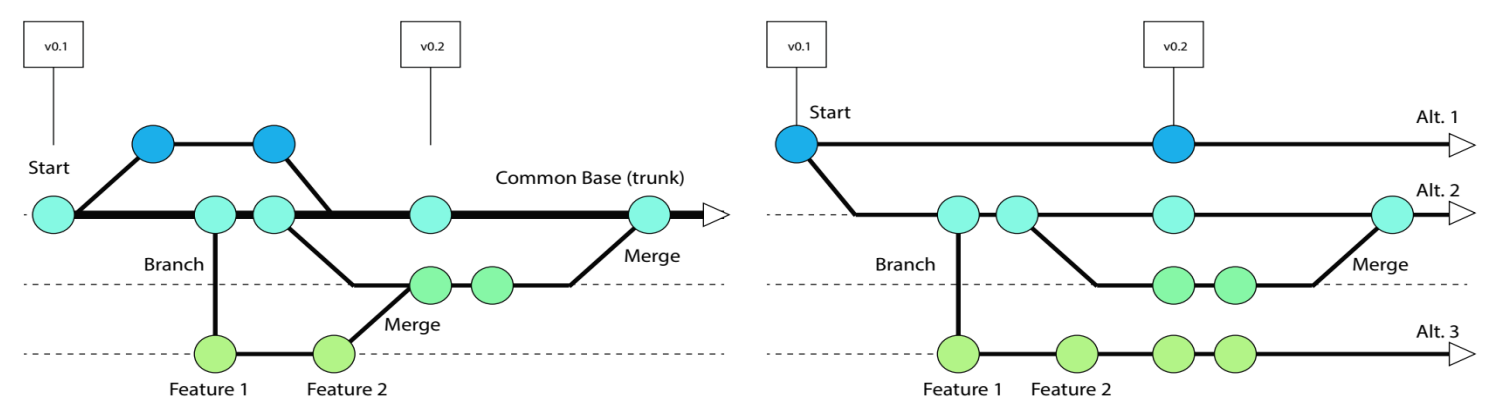

Figure 1. Centralized (on the left) versus Decentralized Version Control (on the right) for Commons-based Peer Production of Shared Knowledge. Illustration by Burak Pak based on cVCS (Stevens 2011).

\section{Critical Analysis of the P2P Facebook Group}

Online creation communities (Fuster Morell 2014) such as Wikipedia and Git interact to build and share knowledge. In this article, however, we analyze a Facebook group because of Facebook's widespread use and implications for democracy (Cadwalladr and Graham-Harrison 2018). Our sample, the P2P Facebook group, has a large number of members $(6,415$ members as of 13 May 2019) and focuses to the commons and P2P (peer to peer). The authors of this article are members of the P2P Facebook group and follow its discussions closely. Self-defined as an 'open contributory forum', P2P Facebook group asks for contributions concerning 'all material that is related to $\mathrm{p} 2 \mathrm{p}$ and the commons' (Anon 2019).

A funneling approach was used for sampling. The data collection was limited to the publicly available data, and no information on user profiles was collected. Researchers were positioned as invisible lurkers (Raymond 2003) and did not contact group admins for more detailed data. This decision prevented us from gathering more detailed data, yet it guaranteed that our data collection process would have no effect on group dynamic. We scanned all posts between 1 April 2018 and 1 April 2019. To examine conflict management and consensus building mechanisms, we selected posts with $100+$ comments for further investigation and examined 26 posts ranging from 100 to 809 comments. Only 6 of these posts contained little or no conflict. 


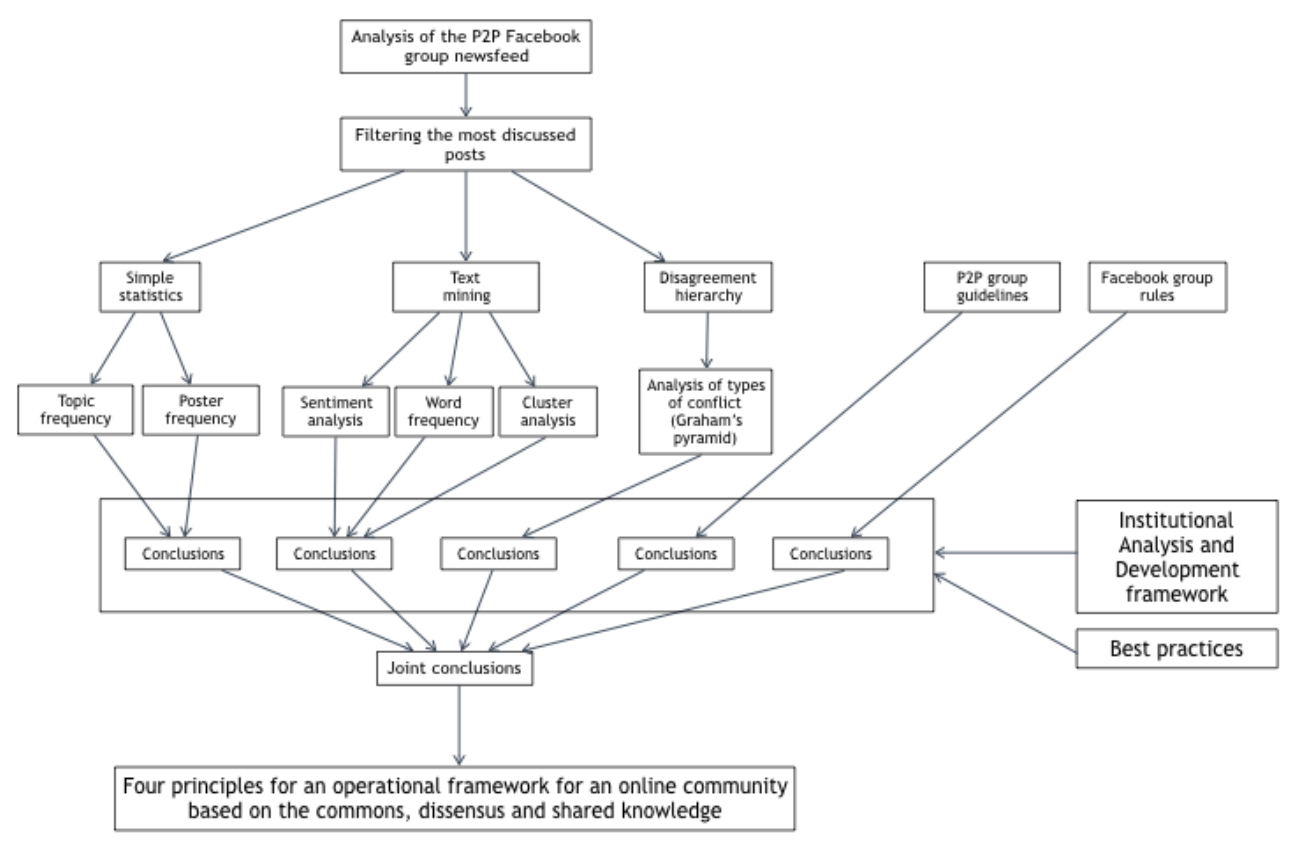

Figure 2. Multiple methods used for the analysis of the P2P Facebook group

\section{Simple Statistics}

The 26 posts with $100+$ comments were analyzed according to original posters, commenters, and discussed topics. 16 were posted by A (A represents one of the founders of P2P Facebook group) and he did not comment on only 3 of the remaining 10 posts. We ran a text analyzer to list the top 20 words in each post. A's name and/or surname was in top 20-word list in 8 out of the 26 posts. Only 2 of the 26 posts were written by women (according to their names and profile pictures). The top 20 -word lists of the 26 posts include only one woman's name. This reveals that women are not only passive in posting on the P2P Facebook group, but they are also not active in commenting. Gender gap in online community participation has been around for some time (Glott, Schmidt, and Ghosh 2010) and the P2P Facebook group has no protocols aimed at encouraging women to participate or be more active in the community. Gender is not the only form of under-representation, but it was the most easily detectable in the scope of this research.

Topics can be grouped in 4 main categories: leadership and moderation in the P2P Facebook group (13 posts), cryptocoins ( 7 posts), political theory (4 posts) and mental illness ( 2 posts). On average the posts about leadership and moderation had the most comments (261), followed by cryptocoins (239), mental illness (227) and political theory (159). Discussions on leadership and moderation can be interpreted as discussions on the protocols. In the triangle of the commons, protocols are shaped and constantly reshaped by the community to share the resources.

\section{Topic}

Average No. of Comments 


\begin{tabular}{ll}
\hline cryptocoins & 239 \\
\hline mental illness & 227 \\
\hline political theory & 159 \\
\hline
\end{tabular}

Table 1. The four main discussion topics in the comments.

Simple statistical analysis brings about following conclusions:

- One of the founders of the P2P Facebook group is over-represented.

- Women are under-represented.

- The community is eager to discuss leadership and moderation (protocols).

Text Mining

In order to systematically analyze discussions, we conducted text mining using RapidMiner and Voyant Tools. A body of 303,463 total words was derived from the posts made between 1 April 2018 and 1 April 2019 that received more than 100 comments.

The first method used was sentiment analysis (also known as opinion mining). This process involved analysis of the corpus and categorization of the sentiment polarity of the terms into positive, negative or neutral expressions using Rapid Miner's AYLIEN Text Analysis extension and its English dictionary. Majority of content was neutral (46.5\%), 29.3\% of terms was positive, and $24 \%$ or terms were negative (Figure 3 ).

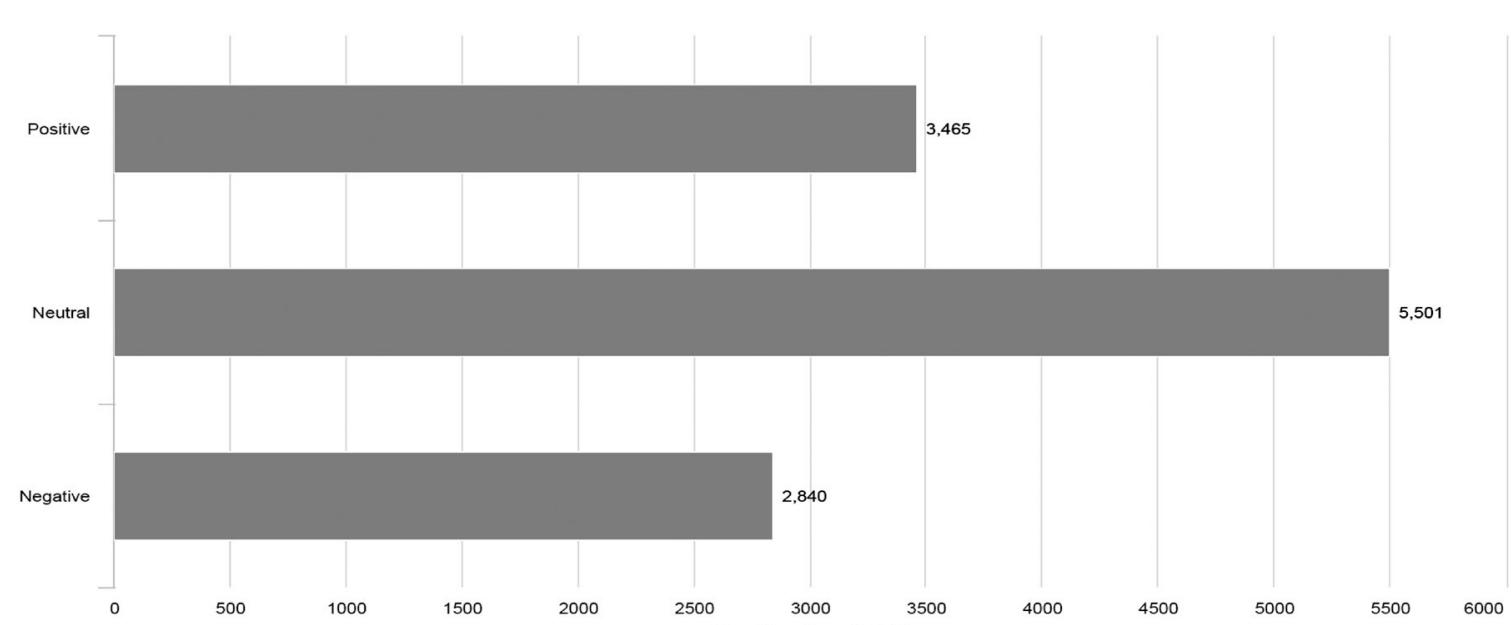

Figure 3. Sentiment analysis of the P2P Facebook group content by using Rapid Miner (11.806 unique word forms).

The second investigation involved the analysis of the most frequent words in the corpus. Figure 4 shows the top 50 terms and their frequencies. The most common word was 'people', 
followed by 'like'. This analysis corresponds to simple statistics analysis, revealing names and surnames of dominant actors and admins: 'Bauwens', 'Nygren', 'Layne', 'Hartsell', and 'Kleiner'. Furthermore, the analysis extracted a total of 458 Internet links.

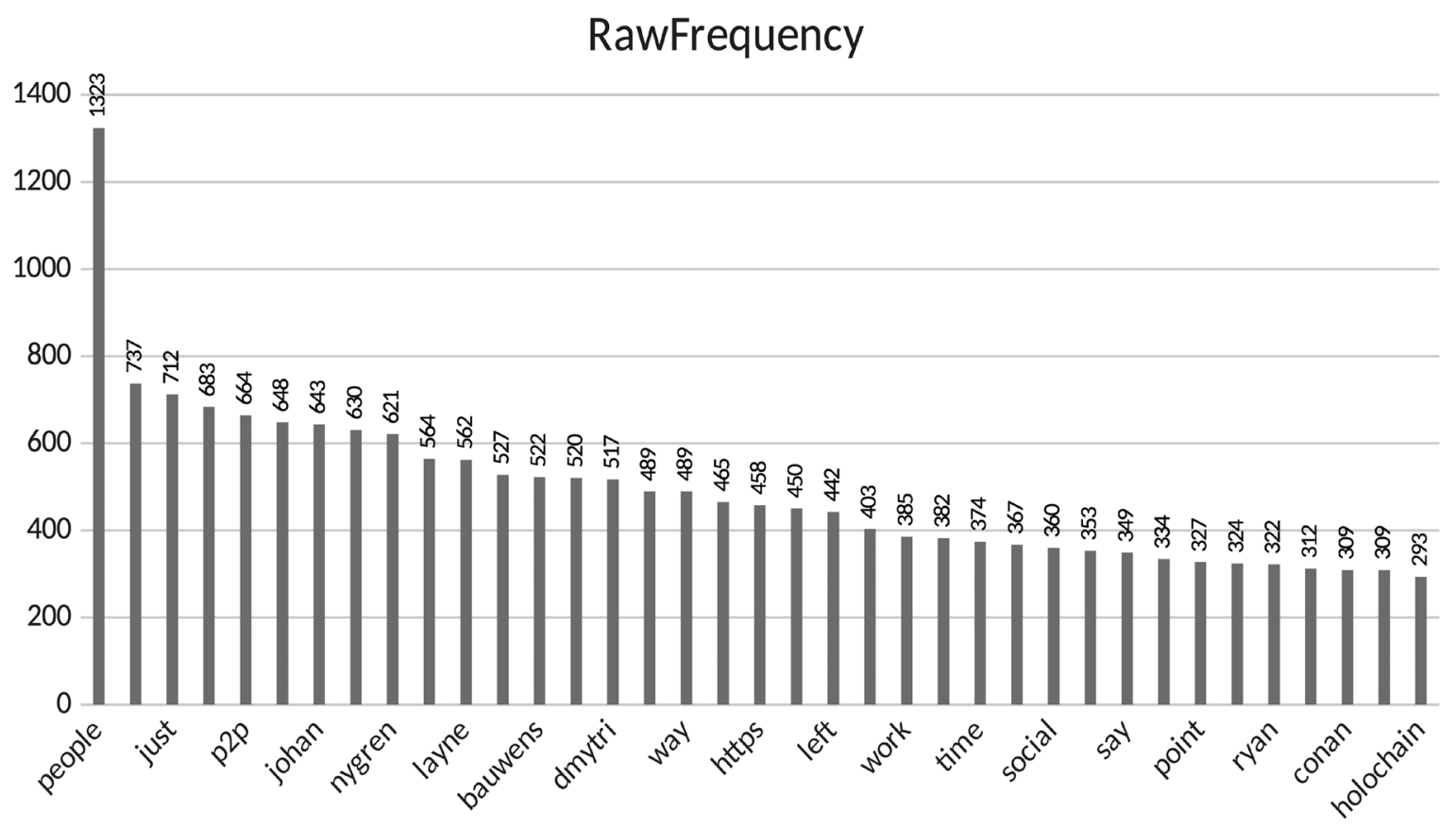

Figure 4. Rapid Miner word count (top 50 terms)

The last method was the Collocates Graph which represents keywords and terms that occur in their close proximity as a force directed network graph. This graph illustrates a network with keywords in green that are shown linked to collocated terms in orange. The size of the terms indicate their frequency and their locations indicate their degree of centrality (Figure 5). Beyond the most frequent words, this graph illustrates the power structure of the P2P Facebook group as well as the most common links and topics discussed.

Text mining brings about following conclusions:

- Only $24 \%$ of content expresses negative sentiments.

- The word 'people' is the most common, followed by 'like' and then by the names of group members.

- People's names are discussed much more frequently than ideas. 


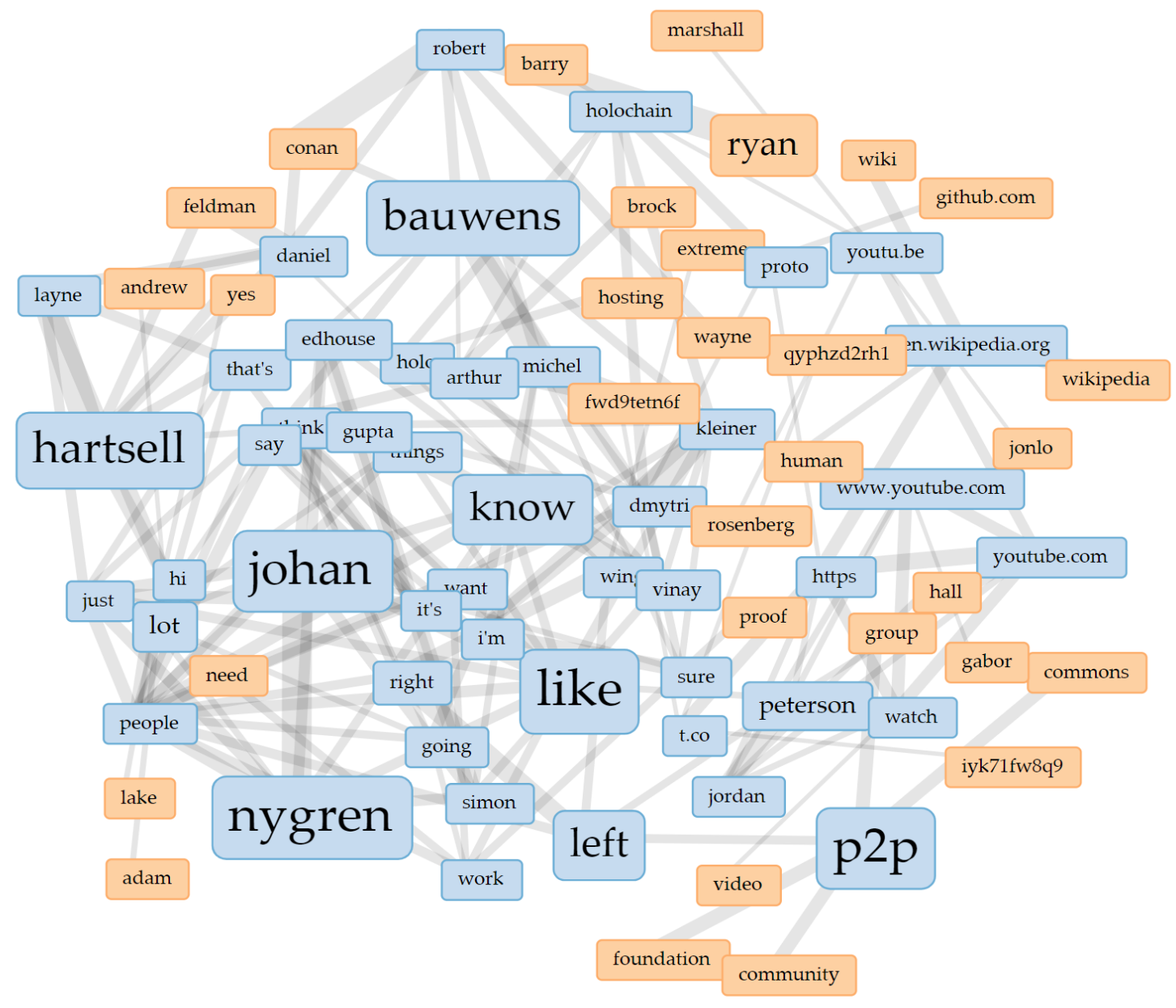

Figure 5. Voyant Tools Collocates Graph of the P2P Facebook group

\section{Disagreement Hierarchy}

Repetition and accumulation of disagreement between people and groups leads to conflict. The aim of disagreement hierarchy analysis is to understand the roots of conflict. Paul Graham's disagreement hierarchy (2008) defines six levels: name-calling, ad hominem, responding to tone, contradiction, counterargument, refutation, and refuting the central point. These levels from name-calling (lowest) to refuting the central point (highest) determine the quality of discussion. We examined the most commented post on leadership and moderation alongside two axes: disagreement hierarchies in people's comments and their definitions of reasons for disagreement. Based on 100 disagreements we detected: 40 ad hominem arguments, 24 responses to tone, 14 contradictions, 17 counterarguments, and 5 refutations. In the P2P Facebook group, talking about the person who comments and how they comment are more frequent than contradicting the views of others with or without reasoning. 


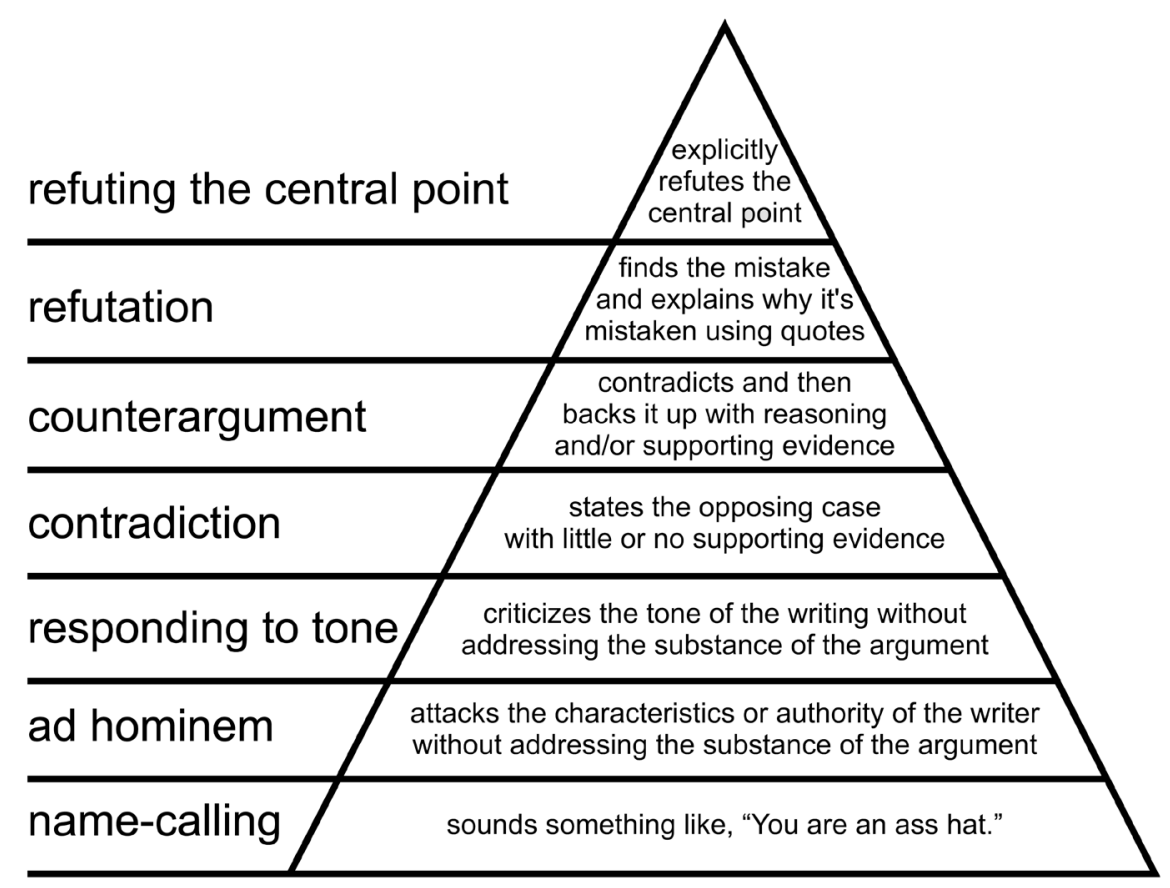

Figure 6. A triangular graphic representing a 'hierarchy of disagreement' from clear refutation to mere vituperation (based on Graham 2008)

\section{P2P Facebook Community Guidelines}

During data collection we encountered an attempt at development of P2P Facebook groupspecific community guidelines. This was penned upon request by one of the members and openly discussed with one of the admins in some threads (P2P Facebook Community Guidelines nd). This link was also available on the P2P Facebook Group under the About section, but it was later removed by its author based on his claims that the admins of the P2P Facebook group were not complying with the community guidelines.

The guideline document has nine sections: P2P Community Agreement (constitutional rules), Admissions (the requirements for becoming a member), Prohibited Actions (how not to engage in interactions), Emergency Deletion (how to delete content), Emergency Suspension (how to cancel memberships temporarily), Official Suspension (how to cancel memberships permanently), Appeals (dealing with objections to suspension or deletion decisions), Adding and Removing Admins (distribution of admin roles), Official Decisions (how to make permanent decisions about admin roles, etc). The document describes the functioning of the admin group in deleting content and accepting or suspending members and/or admins. It is expected for admins to be accountable for their actions to the interested persons, but not to the whole group. For immediate reactions, especially regarding the prohibited actions, admins can act individually, but they have to make more serious decisions (such as permanent suspension or blocking a member, or adding a new admin) collectively.

P2P Facebook group community guidelines bring about following conclusions:

- P2P Facebook group is ruled by an admin group that makes the decisions about group members.

- Administrative decisions are not expected to be explained to the group, but to the members involved through direct communication.

- Admins are expected to react in cases of prohibited actions but not expected to mediate 
milder actions, such as disagreements between members.

\section{Facebook Group Rules}

Facebook group rules can be analyzed on two levels: how groups are situated on the general Facebook platform, and how groups operate autonomously. Ways how Facebook users interact with Facebook group posts are defined by the Facebook News Feed algorithm (Mosseri 2016 and 2018). Following 2016 and 2018 updates, Facebook decided to show more posts from friends and family and less public content such as videos and other posts from publishers or businesses. For example, if you liked the Facebook page of a newspaper and are a part of a Facebook group at the same time, it is more likely you would see the discussions in your Facebook group more frequently than the news links on the newspaper's Facebook page. Facebook's algorithm is also designed to learn from user experiences, and continuous alteration of the algorithm without user control means that, if you are following discussions on a Facebook group, you are likely to see them more often. Autonomous operations of the Facebook groups are regulated with general Facebook policies. In addition to these, Facebook updates group management tools that are also subject to continuous alteration (Deve 2018). There are no specific policies aimed at Facebook groups, neither an explanation of the algorithm defining the way discussions are listed or managed on Facebook groups.

Facebook group rules bring about following conclusions:

- Facebook users have no control or insight over the algorithms that define what they see.

- Facebook algorithms for both news feeds and groups are subject to change according to feedback from user behavior and applied to all users.

- Facebook groups are updated to provide better control over content through admin interference and user complaints in a centralized manner.

\section{Institutional Analysis and Development}

After her comprehensive work on the natural commons Elinor Ostrom developed A Framework for Analyzing the Knowledge Commons with Charlotte Hess. The Institutional Analysis and Development (IAD) framework seeks to understand 'How do fallible humans come together, create communities and organizations, and make decisions and rules in order to sustain a resource or achieve a desired outcome?' (Hess and Ostrom 2007). Simple statistics, text mining, the disagreement hierarchy, and analysis of the P2P Facebook community guidelines and Facebook group rules provide us with variables required by the IAD framework. 


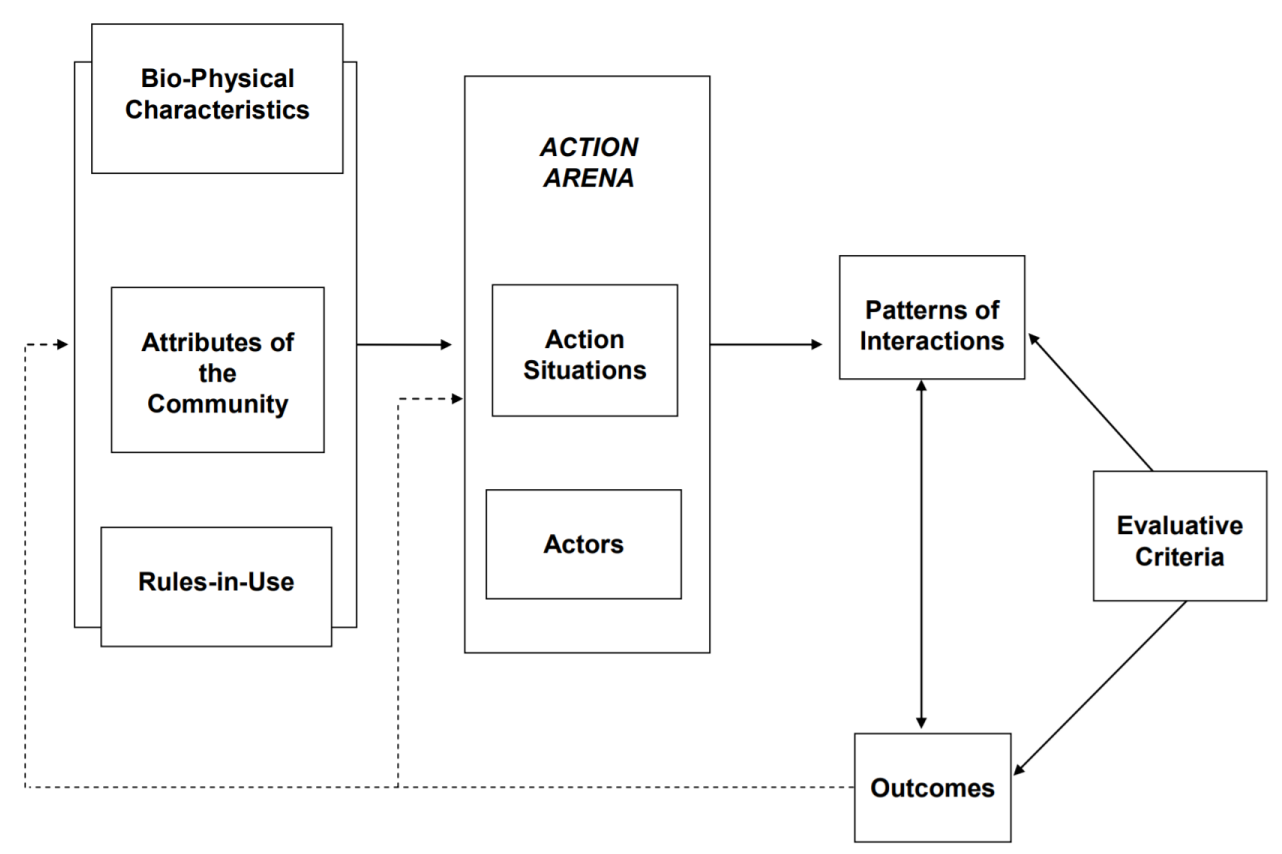

Figure 7. The Institutional Analysis and Development framework (IAD) (Hess and Ostrom 2007)

\section{Resource characteristics}

The P2P Facebook group's bio-physical characteristics include ideas (posts and comments), artifacts (links to websites), and facilities (Facebook server, Internet infrastructure, and the hardware and software used to access to it). The attributes of the community include users (P2P Facebook group members), providers (Facebook) and policymakers (admins in the P2P Facebook group and decision-makers in Facebook). The rules-in-use are operational (P2P Facebook group community guidelines and Facebook policies), collective-choice (P2P Facebook group community guidelines) and constitutional (P2P Facebook group community guidelines). Looking at resource characteristics shows us that some infrastructural parts of the P2P Facebook group are not available for modification by group members or even by group admins. Despite being the main actors, the P2P Facebook group members have no say in defining its rules-in-use.

\section{Action Arena}

Action situations include posting, commenting, liking posts, admitting members, deleting posts, deleting comments, suspending members and blocking members. Actors are defined as members and admins of the P2P Facebook group. Some actions can only be performed by admins (deleting posts and comments, admitting members, and suspending or blocking members) while other actions mostly related to generating content (posting, commenting, etc.) can be done by all members.

\section{Patterns of Interactions}

Drawing from our research we specify three main patterns of interactions that lead to conflicts. Power structures imply hierarchies either constructed with the admin roles or reputations built by frequent contribution. The names of people in these positions are more frequently mentioned 
than other topics of discussion. The community is eager in discussing moderation, which includes power structures. Yet, since admins have control over the group, their decisions are not communicated to the entire group but only to interested parties through private contact. Sentimental behavior concerns feelings during discussions. Most disagreements are caused by referring to people with certain ideas or ideologies not personally, but in a generalized manner. If disagreements are not resolved at the level of ideas, they reoccur and accumulate. While most of the contributions $(46,5 \%)$ are neutral, avoiding to mention main reasons of disagreement also leads to conflict. Grey zones refer to partially known territories of interaction. Even though admins are expected to moderate discussions, their actions are not comprehensible in resolving conflicts between group members. Algorithms that determine which content is to be shown or hidden on Facebook news feed and group feed are not accessible, yet Facebook has communicated its intention to release algorithm updates.

\section{Outcomes}

Outcomes of data analysis include mitotic division (some members leaving the group to start a new one), fluctuation in the number of members, and networking among members.

\section{Evaluative Criteria}

Group's success is defined along the axes of number of members collaborations generated through in-group networking, and knowledge sharing through artifacts (i.e. 458 website links).

\section{Feedback Lines}

IAD framework connects resource characteristics, action arena, patterns of interaction, outcomes, and evaluative criteria, with continuous or dotted lines. These connections, or feedback lines, are necessary for analysis and understanding of relationships between the domains. Feedback is 'the modification or control of a process or system by its results or effects' (Oxford Dictionary 2019). In the P2P Facebook group, there are many criticisms and comments, especially about the patterns of interactions, but they do not affect the group's rulesin-use or outcomes. Therefore, in the P2P Facebook group there is no real feedback as defined in the IAD framework.

Resource characteristics play a significant role in defining the action arena, but the actors (P2P Facebook group members) cannot alter some aspects of the resources (especially the rules-in-use defined by Facebook). Despite the Facebook group members' eagerness to join discussions about moderation and the group's general focus on the topic of $\mathrm{P} 2 \mathrm{P}$ collaboration, the admin role - again defined by Facebook - is primary determinant of patterns of interactions and is not subject to alteration by group members. Generating recurrent conflicts around particular topics without the capability to resolve them - since the admin role cannot be altered or questioned - leads to sentimental behavior. This can be discerned in the high percentage of ad hominem responses and naming people's names more frequently than ideas. Conflict resolution methods are not transparent and visible, but discussions are in place for reaching a consensus about knowledge in the commons. While there is an accumulation of knowledge about the topic, it is vaguely constructed, and it is not clear what the community produces. Shared resources and the community are present, but their protocols are not wholly governed by the community, so a general overview shows that the P2P Facebook group does not function as a commons. 


\section{An Operative Framework for a Novel Online Community Based on Dissensus, the Commons and Shared Knowledge}

This paper critically elaborates dissensual knowledge production as a mechanism for creation and governance of an online knowledge commons. Our discussion about commons and shared knowledge shows the potential of a model of agonistic radical democracy for recognizing and valuing conflict and dissensus, and, in particular, how these can enhance the ways in which commoners create, share and negotiate knowledge as a common pool resource. Inspiring cases for commons-based peer production of shared knowledge are drawn from the Git way of working and distributed version control as innovative collaborative technologies for construction of different realities and results. Our in-depth study of the P2P Facebook group analyzes knowledge production and sharing mechanisms, conflict generation and resolution. It uncovers how a bricolage of truths emerge in the P2P Facebook community. Further discussion of this community based on the Institutional Analysis and Development (IAD) framework shows that infrastructural parts of the P2P Facebook group cannot be managed or modified by group members, and that ordinary members have no say in defining the rules-in-use.

Inspired by these findings, we review commoning practices for knowledge sharing through dissensual collaboration using the IAD framework and identify four key principles: distributed versioning, layering, inclusion and self-determination. These four principles are interlinked and overlap in certain aspects, but we grouped them this way in order to indicate a clear path for the creation of an online community based on commons, dissensus and shared knowledge.

\section{Distributed Versioning}

The idea of versioning is derived from the Git way of working. However, distributed version control systems have also been interpreted in many fields other than software development. They allow knowledge to be produced and accumulated using many tools. Among them, branching, forking and merging are suitable for construction of shared knowledge through dissensual processes. Branching involves interrogating a part of socially constructed truth to add to a main discussion, while forking is cloning the truth to direct it to a different path. Both branching and forking can be merged as a new version or replace a current version. This allows a community to introduce a variety of truths with different frames of reference. What is crucial in this workflow are its distributed characteristics. The control over the version workflow is not centralized in a single person or group, but is distributed among collaborators, allowing for the multiplication of versions. Versions in the context of dissensus are bricolages of truths. Rather than working on conflict resolution to reach consensus, the common goal is defined, and the paths leading to that goal are multiplied according to a community's individual truths.

\section{Layering}

In the distributed version control system, layering makes it possible to conserve all contributions to knowledge production. A centralized version control system, such as the Wiki way of working, is based on compiling all contributions. In its current form, the outcome of a Wiki page is a single entity, and its generation can be seen as version history, making it possible to compare versions. The ideal single entity insists on consensus and singular truth. Layering makes it possible to generate a palimpsest, where different truths are superposed to generate a bricolage of truths, a collective work of knowledge production that can be constructed and deconstructed. The crucial difference between our proposal of layering and the Wiki way of revision history is based on the egalitarian perspective of the commons. No contributions are 
overridden or overwritten by others, and the recognition of the value of multiple voices can create an incentive for further collaboration. This can expand the commoning processes of knowledge production and sharing. Using Graham's (2008) disagreement hierarchy analysis we reveal the importance of the ad hominem disagreements. If consensus is the main goal, conflict is inevitable. However, if we shift the main goal from building consensus to knowledge sharing, then reading a text by peeling through layers of statements by different contributors empowers authorship. This would 'allow differences as differences - all differences - to recognize themselves as constituent power within it.' (Revel 2015)

\section{Inclusion}

Differences among contributors may occur naturally, but their inclusion in commoning processes is defined by existing power structures such as race, gender, and class. 'Resourcerich participants with free time, connectivity, skills and money' (Fuster Morrel 2014) contribute more and are overrepresented in online communities. This claim is also proven by simple statistics, which indicate that women's contribution and visibility is extremely low. We do not have the necessary data collection tools to identify other groups with less accessibility to resources. So, how can we involve them in a dissensual collaboration? One proposed solution is modularity (Benkler and Hessenbaum 2006). Dividing contribution into simple modules such as grammar corrections, adding hyperlinks, and partial editing, allows people with different levels of resources and diverse skill sets to find and invent ways to contribute to knowledge production and sharing. Layering can also enable a community to recognize their contributions, which in turn provides an incentive to continue collaborating.

The second solution lies in a community's engagement with collective-choice arrangements. As our IAD analysis showed, the eagerness of the community to discuss moderation, namely protocols in which they operate is not currently reciprocated by the P2P Facebook group. Group members are not part of the collective-choice arrangements, but their actions are subject to and evaluated by these arrangements. The core of commons thinking requires that the community defines its protocols to share resources. The dynamic characteristics of the commons require these protocols to be altered over time in a form of permanent becoming that allows for differences in the group and also ensures the openness of the commons to include more people.

\section{Self-determination}

The preconditions for a community's self-determination are resource characteristics in the IAD framework. Our case study revealed Facebook's authority to define the protocols of knowledge sharing. These protocols operate in grey zones and cannot be altered by the community. Group members and admins with privileged access to certain features do not have total control over physical infrastructure, data and information. The distributed version control system allows for each user to create their own version repository and store them locally or on own servers. This empowers users to access full content and at the same time protects the community against cooption by centralized corporate structures. The same principles for physical infrastructure can be applied to data, information and knowledge generated by the community, leading to the construction of distributed repositories of dissensus.

\section{Conclusion}

This paper identifies the four key principles that could enhance the democratic practice of online communities: distributed versioning, layering, inclusion and self-determination. The principles rely on tools or practices that already exist in certain communities and online 
platforms (such as Github) and acknowledge the power of the agonistic model where dissensus is accepted as a positive, activating and empowering force. However, the principles open up a number of challenges and issues that require further investigation. First, online platforms, especially those that deal with critical political issues and take normative positions, are not representative of society at large. Users of these platforms have Internet access, are welleducated, know how to navigate and use virtual environments, and are often progressive. An in-depth understanding of the effect of these attributes and skills is outside of the scope of this paper, but their relevance needs to be acknowledged and investigated further.

Second, the drive towards conflict resolution and consensus strongly contradicts Mouffe's agonistic model which sees conflict as a creative, positive force. An agonistic platform requires good protocols to ensure that conflicts and discussions take place at the top of Graham's (2008) hierarchy of disagreement (counter argument, refutation, refuting the central point) and do not regress to lower levels such as name-calling and ad hominem. This concern is closely related to leadership. The role of admins and their engagement with debates needs to embed Freire's dialogical approach (Freire and Horton 1990). For as long as making and remaking of (technical) protocols have not been discussed with all platform users, there can be no neutrality and / or objectivity. Admins cannot take a neutral position. They need to be open about their views and nurture a climate that stimulates dissensus and allows for the existence and emergence of divergent ideas. It is within these restraints, that an operational framework based on the commons, dissensus and shared knowledge developed in this article might provide a useful starting point for developing more open, just, and democratic online communities.

\section{References}

Angermuller, J. (2018). Truth after post-truth: for a Strong Programme in Discourse Studies. Palgrave Communications, 4. https://doi.org/10.1057/s41599-018-0080-1.

Benkler, Y., \& Nissenbaum, H. (2006). Commons-based Peer Production and Virtue. The Journal of Political Philosophy, 14(4), 394-419. https://doi.org/10.1111/j.1467-9760.2006.00235.x.

Blommaert, J. (2016). Het jaar van de informatiecrisis. https://jmeblommaert.wordpress.com/2016/12/18/het-jaar-van-de-informatiecrisis/. Accessed 15 February 2019.

Bodirsky, K. (2018). The commons, property, and ownership. Suggestions for further discussion. Focaal. Journal of Global and Historical Anthropology, 81, 121-130. https://doi.org/10.3167/fcl.2018.810109.

Cadwalladr, C., \& Graham-Harrison E. (2018). Revealed: 50 million Facebook profiles harvested for Cambridge Analytica in major data breach. Guardian, 17 March. https://www.theguardian.com/news/2018/mar/17/cambridge-analytica-facebookinfluence-us-election. Accessed 17 May 2019.

Carpentier, N., \& Cammaerts, B. (2006). Hegemony, Democracy, Agonism and Journalism. Journalism Studies, 7(6), 964-975. https://doi.org/10.1080/14616700600980728.

Chacon, S., Straub, B. (2014). Pro git. https://github.com/progit/progit2. Accessed 14 July 2019.

Dardot, P. (2018). What democracy for the global commons? In S. Cogolati \& J. Wouters (Eds.), The Commons and a New Global Governance (pp. 20-36). Cheltenham (UK): Edward Elgar Publishing. https://doi.org/10.4337/9781788118514.00010.

De Angelis, M., \& Stavrides, S. (2010). On the Commons: A Public Interview with Massimo De Angelis and Stavros Stavrides. E-Flux, 17. June. https://www.e- 
flux.com/journal/17/67351/on-the-commons-a-public-interview-with-massimo-de-angelisand-stavros-stavrides/. Accessed 4 July 2019.

de Tocqueville, A. (1863). Democracy in America. Cambridge: Sever and Francis. https://doi.org/10.2307/2679211.

Description: Welcome to P2P! (n.d.). P2P Facebook Group. https://www.facebook.com/groups/p2p.open/about/. Accessed 14 May 2019.

Deleixhe, M. (2018). Conflicts in common(s)? Radical democracy and the governance of the commons. Thesis Eleven, 144(1), 59-79. https://doi.org/10.1177/0725513618756089

Deve, A. (2018). New Tools to Support Group Admins and Keep Communities Safe. Facebook Newsroom, 23 May. https://newsroom.fb.com/news/2018/05/new-tools-to-support-groupadmins/. Accessed 17 May 2019.

Euler, J. (2018). Conceptualizing the Commons: Moving Beyond the Goods-based Definition by Introducing the Social Practices of Commoning as Vital Determinant. Ecological Economics, 143, 10-16. https://doi.org/10.1016/j.ecolecon.2017.06.020.

Facebook Terms and Policies (n.d.) https://www.facebook.com/policies/. Accessed 16 May 2019.

Foucault, M. (1972). The Archaeology of Knowledge. London: Routledge.

Foucault, M. (1982). The Subject and Power. Critical Inquiry, 8(4), 777-795.

Feedback (n.d.). Oxford Dictionary. https://en.oxforddictionaries.com/definition/feedback. Accessed 19 May 2019.

Fuster Morell, M. (2014). Governance of online creation communities for the building of digital commons: Viewed through the framework of the institutional analysis and development. In M. J. Madison, K. Strandburg, \& B. Frischmann, (Eds.), Governing the Knowledge Commons. New York: Oxford University Press. https://dx.doi.org/10.1093/acprof:oso/9780199972036.003.0009.

Gibson, T. A. (2018). The Post-Truth Double Helix: Reflexivity and Mistrust in Local Politics. International Journal of Communication, 12, 3167-3185.

Gielen, P. (2018). Common Aesthetics: The Shape of a New Meta-Ideology. In N. Dockx \& P. Gielen (Eds.), Commonism: A New Aesthetics of the Real. Amsterdam: Valiz.

Glott, R., Schmidt, P., \& Ghosh, R. (2010). Wikipedia survey - Overview of Results. http://www.ris.org/uploadi/editor/1305050082Wikipedia_Overview_15March2010FINAL.pdf. Accessed 16 May 2019.

Goldstein, D. (1964). Alexis de Tocqueville's Concept of Citizenship. Proceedings of the American Philosophical Society, 108(1), 39-53.

Graham, P. (2008). How to Disagree. http://paulgraham.com/disagree.html. Accessed 12 May 2019.

Hess, C., \& Ostrom, E. (2007). A Framework for Analyzing the Knowledge Commons. In C. Hess \& E. Ostrom (Eds.), Understanding Knowledge as a Commons: From Theory to Practice (pp. 41-82). Cambridge \& London: The MIT Press.

Horton, M., \& Freire, P. (1990). We Make the Road by Walking: Conversations on Education and Social Change. Philadelphia: Temple University Press.

Klein, H. K. (1999). Tocqueville in Cyberspace: Using the Internet for Citizen Associations. The Information Society, 15, 213-220. https://doi.org/10.1080/019722499128376. 
Laclau, E., \& Mouffe, C. (1985). Hegemony and Socialist Strategy. Towards a Radical Democratic Politics. Hegemony and Socialist Strategy. London \& New York: Verso.

McIntyre, L. (2018). Post-truth. Cambridge: MIT Press.

Mosseri, A. (2016). Building a Better News Feed for You. Facebook Newsroom, 29 June. https://newsroom.fb.com/news/2016/06/building-a-better-news-feed-for-you/. Accessed 16 May 2019.

Mosseri, A. (2018). Bringing People Closer Together. Facebook Newsroom, 11 January. https://newsroom.fb.com/news/2018/01/news-feed-fyi-bringing-people-closer-together/. Accessed 16 May 2019.

Mouffe, C. (2018). For a Left Populism. London: Verso.

Olteanu, A., Stables, A., \& Bortun, D. (2019). Meanings \& Co. The Interdisciplinarity of Communication, Semiotics and Multimodality. Cham: Springer.

Ostrom, E. (1990). Governing the Commons. The Evolution of Institutions for Collective Action. Cambridge: Cambridge University Press.

P2P Facebook Community (nd). https://docs.google.com/document/d/1luHJoHx55yF8CUMFQPSoP6NwDU8ocHp9IRar WTa8UsE/edit\#. Accessed 4 July 2019.

Rancière, J. (2009). The Emancipated Spectator. London \& New York: Verso.

Ranciere, J. (2010). Dissensus: On Politics and Aesthetics. London \& New York: Continuum.

Raymond, E. S. (2004). Lurker. The online hacker Jargon File, version 4.4.7. http://catb.org/jargon/html/L/lurker.html. Accessed 16 May 2019.

Revel, J. (2015). Producing Subjectivity, Producing the Common: Three challenges and a slightly long postscript on what the common is not. In V. Bobin (Ed.), Composing Differences. Dijon: Les Presses du Reél.

Stevens, A. (2011). Distributed Version Control Systems: A Guide For The Perplexed. Slideshare, 13 October. https://www.slideshare.net/alanstevens/distributed-version-control-withmercurial. Accessed 25 May 2019.

Susser, I. (2017a). Commoning in New York City, Barcelona, and Paris. Focaal. Journal of Global and Historical Anthropology, 79, 6-22. https://doi.org/10.3167/fc1.2017.790102.

Susser, I. (2017b). Introduction. For or against commoning ? Focaal. Journal of Global and Historical Anthropology, 79, 1-5. https://doi.org/10.3167/fcl.2017.790101.

Temmerman, M., Moernaut, R., Coesemans, R., \& Mast, J. (2019). Post-truth and the political : Constructions and distortions in representing political facts. Discourse, Context \& Media, 27, 1-6. https://doi.org/10.1016/j.dcm.2018.10.002.

Torvalds, L. (2007). Tech talk: Linus Torvalds on git. Google Tech Talk Conference.https://www.youtube.com/watch?v=4XpnKHJAok. Accessed 14 July 2019.

\section{Acknowledgments}

This paper is co-created as part of a Ph.D. research project by Hülya Ertas 'Building a Knowledge Commons for Commons Architecture' at the KU Leuven Department of Architecture, Campus Sint-Lucas Brussels, co-advised by Burak Pak, Caroline Newton and Lieven de Cauter. The online platform proposed in this chapter will be implemented as a part of her Ph.D. project.

We would like to thank our editor Petar Jandrić and anonymous reviewers for their constructive feedback and Thomas Glenn Kelso for the proofreading. 